\title{
Scale and Rotation Invariant Detection of Singular Patterns in Vector Flow Fields
}

\author{
Wei Liu and Eraldo Ribeiro \\ Computer Vision and Bio-Inspired Computing Laboratory \\ Department of Computer Sciences \\ Florida Institute of Technology \\ Melbourne, FL 32901, USA \\ $\{$ lwei,eribeiro\}@fit.edu
}

\begin{abstract}
We present a method for detecting and describing features in vector flow fields. Our method models flow fields locally using a linear combination of complex monomials. These monomials form an orthogonal basis for analytic flows with respect to a correlation-based innerproduct. We investigate the invariance properties of the coefficients of the approximation polynomials under both rotation and scaling operators. We then propose a descriptor for local flow patterns, and developed a method for comparing them invariantly against rigid transformations. Additionally, we propose a SIFT-like detector that can automatically detect singular flow patterns at different scales and orientations. Promising detection results are obtained on different fluid flow data.
\end{abstract}

\section{Introduction}

Detecting patterns in vector flow fields is key to many computer vision and engineering applications including texture analysis [16, fingerprint classification [6 14], and fluid dynamics 417]. In principle, singular flow-pattern detection is similar to the interest point detection problem in scalar images [13. However, the number of flow-field descriptor approaches in the computer vision literature is relatively limited. In this paper, we introduce a novel scale-rotation invariant framework for detecting singular patterns in vector flow data.

Vector field data usually originate from continuous physical processes such as motion and dynamic textures. As a result, model-based approaches for singular pattern detection are common in the literature. For example, template-matching approaches using correlation [17] or filtering operations [14] are generally robust. However, pattern detected by these approaches are often restricted to the template's size and shape. Another class of singular-pattern detection methods are based on locally-affine flow-field models [16]. An extension to a nonlinear flow model was proposed by Ford et al. [8, and most recently Kihl et al. [1] improved it further to detect multi-scale singular points. Finally, flow fields can also be represented using complex functions. For instance, Fan et al. [6] used the complex zero-pole model to detect singular points in fingerprint images. An earlier work by Nogawa et al. [15] modeled singular patterns based on Cauchy's residue 
theorem. Nevertheless, residue calculation can be sensitive to noise. Corpetti et al. 4 detected singular-flow patterns as the maxima of complex potential and streamline functions that were obtained from flow fields' irrotational and solenoidal components. Corpetti's detected singular patterns were quite general as they did not need to contain a center vanishing point.

In this paper, we propose a novel framework for the detection and description of singular patterns in vector fields under rigid transformations (i.e., rotation and scale invariant). We commence by approximating the flow field locally using a linear combination of complex analytic basis functions (Section 2). We use the approximation coefficients as a flow descriptor. Our selected set of complex basis functions can be shown to be eigenfunctions of the rotation operator. This observation allows us to define a concept equivalent to a flow pattern's principle orientations by aligning it to the analytic bases. We will show that scaling a flow field corresponds to scaling our descriptor. By aligning descriptors using the estimated principle orientations, and normalizing them in scale, flow patterns can be directly compared (Section 3). Finally, we introduce a multi-scale singular pattern detector (Section 4). As in [4, we are able to detect singular patterns in a broader sense than the commonly used vanishing singular points. Our experimental results (Section 5) demonstrate the effectiveness of our descriptor by both detecting and clustering singular patterns on various flow field sequences.

\section{Higher-Order Model of Flow Field}

We begin by representing a $2-\mathrm{D}$ vector-flow field as a complex-valued function $F(z)$ defined on a finite domain $\Omega \subset \mathbb{C}$. Locally, a flow field can be represented by an analytic function centered at $z_{0} \in \mathbb{C}$, i.e., $f(z) \approx F\left(z+z_{0}\right) \square$ The Taylor expansion of $f(z)$ about the origin (i.e., $z_{0}=0$ ) can be written as a linear combination of complex (orthogonal) basis functions $\phi_{k}(z)$ as follows:

$$
f(z)=\sum_{k=0}^{N} a_{k} \phi_{k}(z)+R_{N}(z),
$$

where $a_{k}=\frac{f^{(k)}(0)}{k !}$ are the coefficients, and $R_{N}(z)$ is the residue. Here, $f^{(k)}(0)$ is the $k$-th derivative of $f$ evaluated at $z_{0}=0$. There are number of choices of polynomial bases $\phi_{k}(z)$ that are equivalent from both the functional analysis and approximation theory viewpoints, e.g., complex-domain Zernike polynomials 10, and real-domain Legendre polynomials [11. Our goal is to approximate flow fields locally. While this goal can be accomplished using linear models based on real-domain basis functions [1116], we believe that complex functions are valuable bases to model smooth natural motions [10 154]. Additionally, complex bases are usually more compact than their real orthogonal counterparts.

${ }^{1}$ The analytic assumption was also used in 154 . While theoretically some linear flow fields are not analytic, they can be considered less physically relevant [4]. 
It is worth pointing out that both the orthogonality condition and basisfunction projection depend on the choice of inner product in the analytic functions space $A(\Omega)$ on $\Omega$. Using the standard inner product defined for complex functions in $\mathbf{L}^{2}$ [5] results in complex numbers, making projection calculations difficult. Instead, we adopt the following alternative inner product:

$$
\langle f(z), g(z)\rangle=\int_{\mathbb{C}} f(z) \cdot g(z) d z
$$

where $\cdot$ is the standard inner product on $\mathbb{C}$ (i.e., dot product between two complex numbers). Equation[2] satisfies the three inner product axioms [5]: symmetry, linearity, and positive-definiteness, and it can be used to project flow field $f(z)$ onto the basis function $\phi_{k}(z)$, with real-domain projection coefficients given by $a_{k}=\frac{\left\langle f(z), \phi_{k}(z)\right\rangle}{\left\langle\phi_{k}(z), \phi_{k}(z)\right\rangle}$. Furthermore, we can re-write Equation 2 as:

$$
\langle f(z), g(z)\rangle=(F \otimes g)\left(z_{0}\right)=\int_{\mathbb{C}} F\left(z+z_{0}\right) \cdot g(z) d z,
$$

which is similar to the cross-correlation operator used in [17, and can be implemented efficiently using the Fast Fourier Transform (FFT).

In this paper, we use the complex-domain monomials $\left\{z^{k}\right\}_{k=1}^{N}$ as basis functions $\phi_{k}(z)$. We can show that $i z^{k}$ belongs to the same basis formed by $z^{k}$, and that the basis is complete. Intuitively, $i z^{k}$ can be thought as a counterclockwise 90-degree rotation of the vectors in $z^{k}$. Without affecting the orthogonality of $z^{k}$ and $i z^{k}$, we control the basis' local support size by weighting the basis with a zero-mean Gaussian function $w_{\sigma}(y)$. Our basis flows can then be written as:

$$
\phi_{k, 1}(z)=\frac{z^{k} w_{\sigma}(z)}{\left\|z^{k} w_{\sigma}(z)\right\|} \quad \text { and } \quad \phi_{k, 2}(z)=\frac{i z^{k} w_{\sigma}(z)}{\left\|i z^{k} w_{\sigma}(z)\right\|},
$$

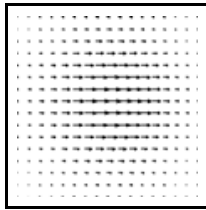

(a) $\phi_{0,1}$

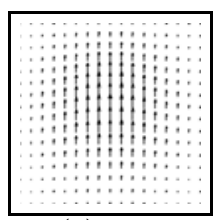

(e) $\phi_{0,2}$

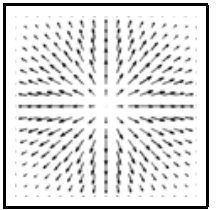

(b) $\phi_{1,1}$

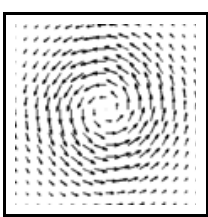

(f) $\phi_{1,2}$

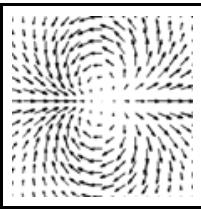

(c) $\phi_{2,1}$

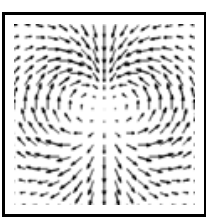

(g) $\phi_{2,2}$

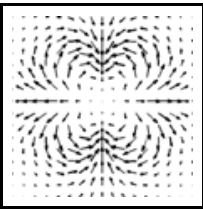

(d) $\phi_{3,1}$

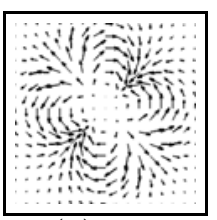

(h) $\phi_{3,2}$

Fig. 1. Basis flows $\phi_{k, i}, k=0, \ldots, 3$ and $i=1,2$. Row 1: polynomials derived from $z^{k}$. Row 2: polynomials derived from $i z^{k}$. Increasing $k$ produce higher-order fluctuations. 


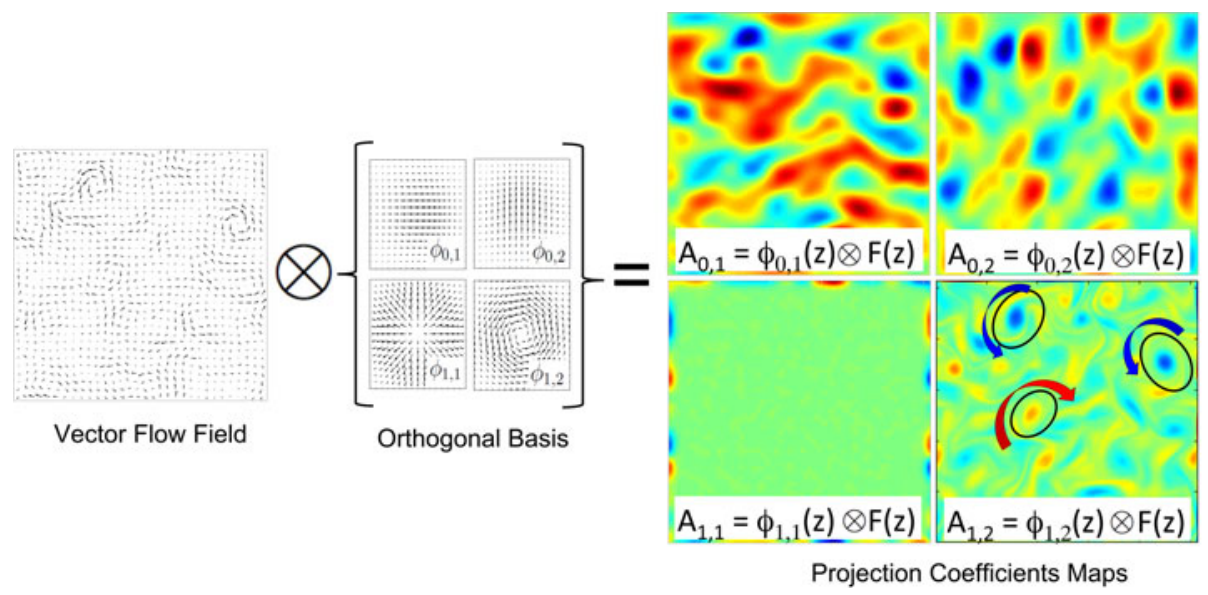

Fig. 2. Cross-correlation between the flow field and the first four bases $\phi_{k, 1}(z)$. Map $A_{1,1}$ indicates a divergence-free flow field. Peaks in $A_{1,2}$ indicate vortices. Blue: matching orientation between filter and flow data. Red: reverse orientation.

where $\|\phi\|^{2}=\langle\phi, \phi\rangle$. The orthonormal basis $\phi_{k, i}$ for $k=0,1,2,3$ are shown in Figure 1. Using (1), the $N$-th order flow field approximation at $p \in \Omega \subset \mathbb{C}$ is:

$$
F\left(z+z_{0}\right) \approx f(z)=\sum_{k=0}^{N}\left[a_{k, 1} \phi_{k, 1}(z)+a_{k, 2} \phi_{k, 2}(z)\right],
$$

where $a_{k, i}=\left\langle f(z), \phi_{k, i}(z)\right\rangle$, for $k=1, \ldots, N$, and $i=1,2$. The approximation produces $2(N+1)$ real coefficients $a^{p}=a_{0,1}^{p}, a_{0,2}^{p}, \ldots, a_{N, 1}^{p}, a_{N, 2}^{p}$ for location $p$. According to (3), the coefficients are local values of the cross-correlation between $F(z)$ and $\phi_{k, i}(z)$. Figure 2 shows the correlation of the first two basis pairs with a turbulent flow, i.e., $A_{k, 1}=F(z) \otimes \phi_{k, 1}(z)$ and $A_{k, 2}=F(z) \otimes \phi_{k, 2}(z), k=0,1$.

\section{Flow Field Descriptor}

In the previous section, a local approximation of local flow fields was presented. We will now show how the projection coefficients can be used to derive descriptors that are invariant to both rotation and scaling transformations.

The rotation operator. Let us consider the flow-field expansion given by (5). The rotation operator $\Gamma_{\theta}(\cdot)$ rotates the flow $f(z)$ by an angle $\theta$ as follows:

$$
\begin{aligned}
\Gamma_{\theta}(f(z))=e^{-\theta i} f\left(z e^{\theta i}\right) & =e^{-\theta i} \sum_{k=0}^{N}\left[a_{k, 1} \phi_{k, 1}\left(z e^{\theta i}\right)+a_{k, 2} \phi_{k, 2}\left(z e^{\theta i}\right)\right] \\
& =\sum_{k=0}^{N}\left[a_{k, 1} \Gamma_{\theta}\left(\phi_{k, 1}(z)\right)+a_{k, 2} \Gamma_{\theta}\left(\phi_{k, 2}(z)\right)\right] .
\end{aligned}
$$


Here, $e^{-\theta i}$ is the contravariant factor to ensure coordinate invariance of the vector field [1. $\Gamma_{\theta}$ is linear on the analytic function space $\mathbb{A}^{n}$. Furthermore, our choice of basis monomials, $z^{k}$ and $i z^{k}$, are eigenfunctions for $\Gamma_{\theta}$, i.e.,

$$
\Gamma_{\theta}\left(z^{k}\right)=e^{(k-1) \theta i} z^{k} \quad \text { and } \quad \Gamma_{\theta}\left(i z^{k}\right)=e^{(k-1) \theta i} i z^{k},
$$

with eigenvalues equal to $e^{(k-1) \theta i}$. The bases' Gaussian weighting and the normalizing constant in (4) are rotation invariant so they were dropped. By plugging (17) into (6) and re-arranging the basis monomial terms, we obtain:

$$
\begin{aligned}
& a_{k, 1}^{\prime}(\theta)=\cos [(k-1) \theta] a_{k, 1}-\sin [(k-1) \theta] a_{k, 2} \\
& a_{k, 2}^{\prime}(\theta)=\sin [(k-1) \theta] a_{k, 1}+\cos [(k-1) \theta] a_{k, 2},
\end{aligned}
$$

where $a_{k, i}$ and $a_{k, i}^{\prime}$ are the coefficients for the original and rotated flow fields, respectively. Equation 8 shows that rotating a flow field also rotates their projection coefficients. Our goal is to compare flows by rotating the coefficients to a standard orientation. However, the rotation angle is unknown. We solve this problem by finding the angle that maximizes the alignment between the local flow and a subset of our eigenfunctions that are not rotationally symmetric (i.e., except $z$ and $i z$ ), and calculate $\theta$ that maximizes the inner-product projection:

$$
\widetilde{\theta}=\underset{\theta}{\arg \max } \sum a_{k, 1}^{\prime}(\theta) .
$$

The above maximization of a trigonometric polynomial function can be solved using standard optimization algorithms (e.g., Gauss-Newton method). Computationally, rotating the coefficients is far more efficient than rotating the flow field itself. We call the values of $\theta$ at these local maxima the Principle Orientations. Once these directions are at hand, we can compare two flow fields, $f_{p}(z)$ and $f_{q}(z)$, by defining a distance between them. We use the minimum Euclidean distance between their rotated coefficients defined as follows:

$$
d\left(f_{p}, f_{q}\right)=\min _{i, j}\left\|\Gamma_{\theta_{i}} a_{p}-\Gamma_{\theta_{j}} a_{q}\right\| .
$$

Vector fields' directional nature generate multiple principle orientations, and Equation 9] has at most $2 N$ roots [9]. Rather than finding the "best" orientation, we accept all principle orientations for which this equation exceeds a threshold.

The scaling operator. Let us now consider the scaling operator $\Psi_{s}(),. s>0$ applied on the weighted basis flow defined in (4). This operator is also linear, and scaling effects are fully defined on the basis functions $\phi_{k, i}$ as follows:

$$
\begin{aligned}
\Psi_{s}\left(\phi_{k, 1}(z)\right) & =s \phi_{k, 1}\left(s^{-1} z\right)=s \frac{\left(s^{-k}\right)}{\left|\left(s^{-k}\right)\right|} \frac{z^{k} w_{\sigma}\left(s^{-1} z\right)}{\left\|z^{k} w_{\sigma}\left(s^{-1} z\right)\right\|} \\
& =s \frac{z^{k} w_{\sigma}\left(s^{-1} z\right)}{\left\|z^{k} w_{\sigma}\left(s^{-1} z\right)\right\|}=s \frac{z^{k} w_{s \sigma}(z)}{\left\|z^{k} w_{s \sigma}(z)\right\|}
\end{aligned}
$$


Therefore, scaled bases can be obtained by scaling the variance of the Gaussian weighting function, and then multiplying them by $s$. The relationship holds for both $z^{k}$ and $i z^{k}$ bases. Next, we use these ideas to develop a method for detecting interest flow patterns under scaling and rotation transformations.

\section{Detection of Singular Patterns}

Singular points (or critical points) in vector fields can be defined as locations where the flow field vanishes [116]7, i.e., $F(z)=0$. If we consider the expansion in Equation 5, then $F(z)=0$ implies $a_{0,1}=a_{0,2}=0$. As a result, a local flow pattern containing a singular point at the center can be linearly approximated by $\phi_{k, i}$ with $k \geq 1$. We will name $\phi_{k, i}, k \geq 1$ the singular basis, and will assume that the flow field's linear expansion can be separated into two components: the background constant flow expanded by $\phi_{0,1}, \phi_{0,2}$, and a singular component expanded by the singular basis. The constant flow is similar to the laminar component mentioned in 4]. We define a singular point as maxima of the flow field energy projected onto the singular basis. The singular energy function is defined as the squared sum of projection coefficients on the singular basis, i.e., $E_{\text {sig }}(z)=\sum_{k=1}^{N}\left(\left\|a_{k, 1}\right\|^{2}+\left\|a_{k, 2}\right\|^{2}\right)$. As in [4], the separation of background constant flow makes our definition of singular patterns more general than the singular points defined in [11167], since a flow field may not have any vanishing points when a background constant flow or a laminar flow exists.

Comparing flows of similar sizes can be achieved by using Equation 10. We now look into the case of detecting singular patterns at multiple scales. Here, we will approach the multiple-scale problem in a similar way as done in scale-space theory for scalar images. We begin by applying a Gaussian smoothing to the vector field followed by a down-sampling operation [13. In the case of vector fields, the Gaussian smoothing step might actually destroy singular points [7. Using the properties described in Section 3, we keep the flow field unchanged,

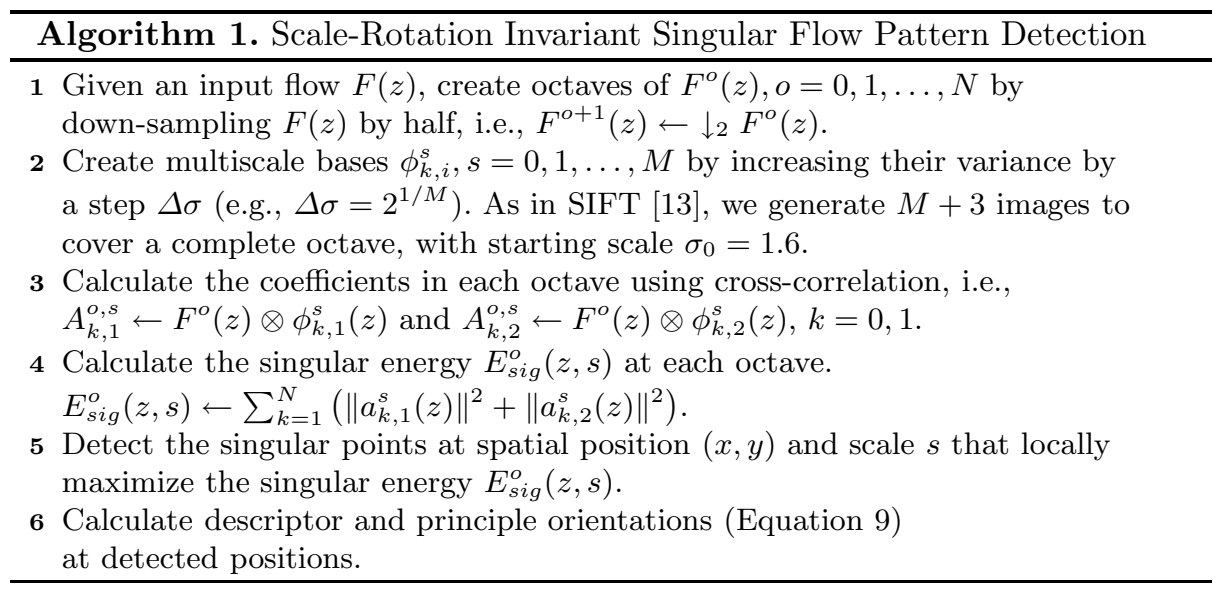


and instead vary the scale of the basis function. Scaling the basis function only involves changing the variance parameter of the Gaussian weighting function, and it does not destroy the singular points. However, increasing the basis flow size increases the computation due to the correlation operation in Equation 3 .

To address these problems, we adopt a hybrid method for multiscale singularpattern detection. Similarly to the SIFT descriptor [13, we divide the scale space into octaves using Gaussian smoothing and down-sampling. However, scaling is applied to the basis flows within each octave. Singular pattern candidate scales are selected as extrema of singular energy $E_{\text {sig }}$ along both the scale and spatial dimensions. Algorithm 1 summarizes the detection process.

\section{Experiments}

We tested our detector on sequences from European FLUID Project [3], and satellite imagery obtained from the SSEC Data Center 2 . Additionally, we tested the flow descriptor by automatically clustering singular patterns of varying scale and orientation that were extracted from the JHU Turbulence dataset [12].

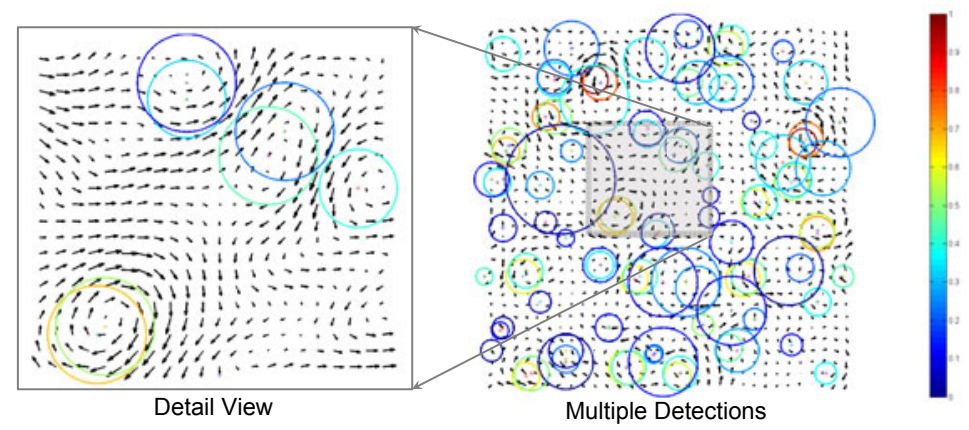

Fig. 3. Right: detected patterns. Color indicates the relative log magnitude of singular energy. Vortices are the strongest patterns; Left: detail view of detected patterns.

Detection on FLUID sequences. Detected singular patterns from a FLUID sequence are shown in Figure 3, The patterns' singular energy was color-mapped for visualization clarity. This dataset contains sourceless vector fields, and most singular patterns resemble vortices appearing at multiple scales. Our method detected all vortices. Elongated-shaped vortices were detected in pairs. In these cases, some detections could have been discarded based on their singular energy.

Detection on satellite images. In this experiment, we extracted velocity field data from SSEC satellite image sequences using the CLG optical-flow algorithm [2]. CLG produced fairly good estimation results considering that accurate

2 http://www.ssec.wisc.edu/data/us_comp/ 

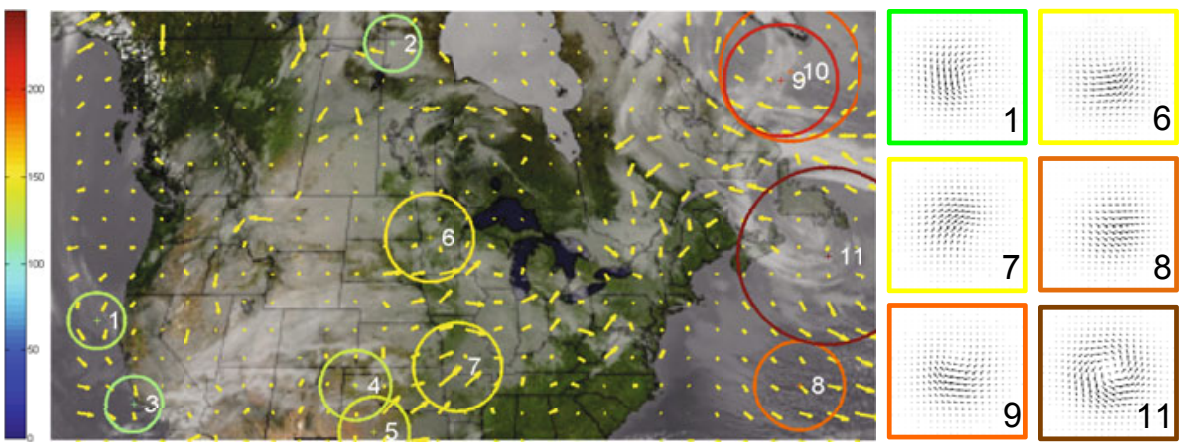

Fig. 4. Singular pattern detection in satellite image sequence. Estimated flow field is downsampled for visualization. Strong patterns to the north-east $(9,10,11)$ corresponds to vortices. South-east singular pattern (8) corresponds to sudden clouds divergence.

fluid-motion estimation is not our method's main focus. Detection results produced by our detector on motion clouds are shown in Figure 4. The figure shows a satellite image of a U.S. weather system on February 20th, 2010. For better visualization, singular patterns smaller than 20 pixels in diameter were removed. On the northeast corner, large vortices were detected. On the southeast corner, a strong singular pattern corresponds to clouds disappearance and divergence. Most detected patterns are consistent with cloud motion changes.

Detecting and Clustering. In this experiment, we clustered singular patterns detected on the JHU 3-D Turbulence dataset. Here, we selected 2-D slices that were perpendicular to the flow's convecting direction. For better visualization, we created two groups of detected patterns according to their similarity to vortices and sources (or sinks). We did that by examining whether the singular energy was concentrated on the basis functions $\phi_{1,1}, \phi_{1,2}$. If $\left\|a_{1,1}\right\|^{2}+\left\|a_{2,2}\right\|^{2}$ consisted of more than $60 \%$ of the total singular energy, then we labeled the singular pattern as symmetric, otherwise, we call it asymmetric.

We then scaled and aligned the features. For patterns having multiple principle orientations, we generated multiple aligned copies, and created four groups using k-means. Clusters for symmetric features are shown in Figure 5 (top), while clusters of asymmetric features are shown in Figure [5](bottom). Symmetric patterns mostly corresponded to vortices in both directions, sources, and swirls. Due to the flow's divergent nature, few sinks were detected, and no sink clusters were obtained. Asymmetric patterns mostly correspond to vortices skewed by a background laminar. Clusters were distinguished by their rotation direction, and their divergence or convergence. Most patterns in this group did not have a center vanishing point, yet they still exhibited interesting sudden flow field changes. This suggests the generality of our singular pattern definition. 


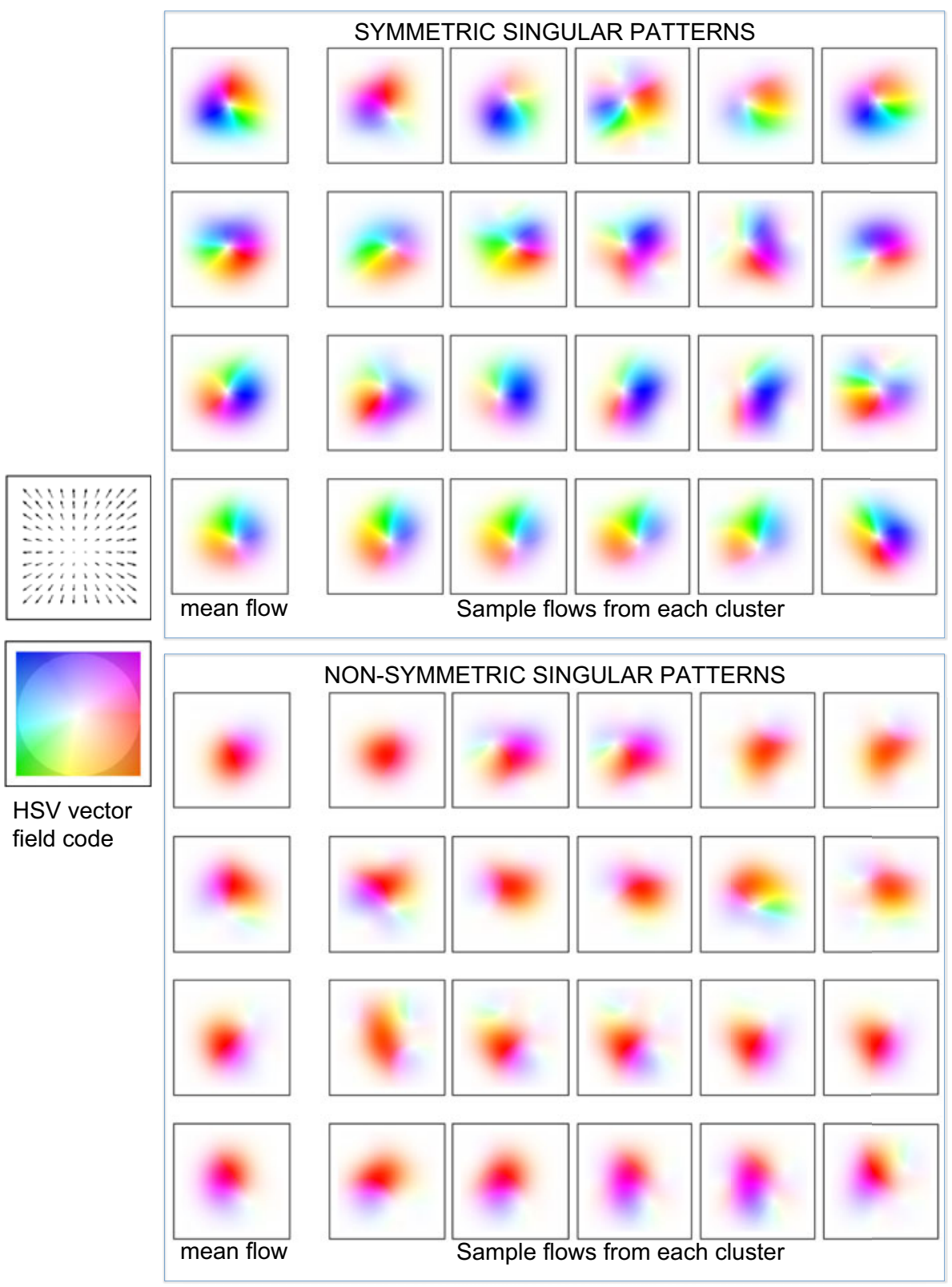

Fig. 5. Clusters of symmetric and asymmetric singular patterns detected on the JHU 3-D Turbulence dataset. Each row displays cluster means and sample flows. 


\section{Conclusion}

We proposed a flow-field descriptor based on coefficients of a local flow field approximation. Based on this descriptor, we designed a SIFT-like detector for singular patterns that is invariant to rigid transformations. The detector was tested on both synthetic and real fluid flows. Future work includes an extension to 3-D flow fields and exploring new applications.

\section{References}

1. Bronshtein, I., Semendyayev, K., Musiol, G., Muehlig, H.: Handbook of mathematics. Springer, Berlin (1997)

2. Bruhn, A., Weickert, J., Schnörr, C.: Lucas/Kanade meets Horn/Schunck: combining local and global optic flow methods. Int. J. Comp. Vis. 61(3), 211-231 (2005)

3. Carlier, J.: Second set of fluid mechanics image sequences. In: European Project 'Fluid image analysis and description, FLUID (2005), http://www.fluid.irisa.fr/

4. Corpetti, T., Mémin, E., Pérez, P.: Extraction of singular points from dense motion fields: An analytic approach. J. Math. Imaging Vis. 19(3), 175-198 (2003)

5. Davies, B.: Integral Transforms and Their Applications. Springer, Heidelberg (2002)

6. Fan, L., Wang, S., Wang, H., Guo, T.: Singular points detection based on zero-pole model in fingerprint images. Trans. Patt. Anal. Mach. Intell. 30(6), 929-940 (2008)

7. Florack, L.: Scale-space theories for scalar and vector images. In: Scale-Space 2001, London, UK, 2001, pp. 193-204. Springer, London (2001)

8. Ford, R.M., Strickland, R.N., Thomas, B.A.: Image models for 2-D flow visualization and compression. Graph. Models Image Process. 56(1), 75-93 (1994)

9. Forray, M.J.: Approximation Theory and Methods. Cambridge Univ. Press, Cambridge (1981)

10. Hoey, J., Little, J.J.: Bayesian clustering of optical flow fields. ICCV 2, 1086 (2003)

11. Kihl, O., Tremblais, B., Augereau, B.: Multivariate orthogonal polynomials to extract singular points. In: ICIP, pp. 857-860 (2008)

12. Li, Y., Perlman, E., Wan, M., Yang, Y., Meneveau, C., Burns, R., Chen, S., Szalay, A., Eyink, G.: A public turbulence database cluster and applications to study Lagrangian evolution of velocity increments in turbulence. Journal of Turbulence 9(31), 1-29 (2008)

13. Lowe, D.G.: Distinctive image features from scale-invariant keypoints. Int. J. Comput. Vision 60(2), 91-110 (2004)

14. Nilsson, K., Bigun, J.: Localization of corresponding points in fingerprints by complex filtering. Pattern Recogn. Lett. 24(13), 2135-2144 (2003)

15. Nogawa, H., Nakajima, Y., Sato, Y., Tamura, S.: Acquisition of symbolic description from flow fields: a new approach based on a fluid model. IEEE Trans. Patt. Anal. Mach. Intell. 19(1), 58-63 (1997)

16. Rao, A.R., Jain, R.C.: Computerized flow field analysis: Oriented texture fields. IEEE Trans. Pattern Anal. Mach. Intell. 14(7), 693-709 (1992)

17. Schlemmer, M., Heringer, M., Morr, F., Hotz, I., Hering-Bertram, M., Garth, C., Kollmann, W., Hamann, B., Hagen, H.: Moment invariants for the analysis of 2D flow fields. IEEE Trans. on Vis. and Comp. Graph 13(6), 1743-1750 (2007) 